\title{
ALZHEIMER'S COGNITIVE IMPAIRMENT CAN BE CURED BY DECREASING BLOOD HOMOCYSTEIC ACID
}

\author{
T. Hasegawa ${ }^{1}$, T. Uchida ${ }^{1}$, A. Chanoki
}

\begin{abstract}
Recent big two clinical trials of amyloid beta treatment for Alzheimer's disease are all failed to recover the cognitive impairment, it has forced us to reconsider the central hypothesis of amyloid pathogen for Alzheimer's disease. We recently published that human Alzheimer's patients showed the strong relationship between Mini Mental State Examination (MMSE) scores and blood homocysteic acid (HA) level. However this relationship did not show the pathogenic action of HA. So we investigated the pathogenic effect of blood HA on MMSE score, that is, how does blood HA level change affect MMSE score? 6 AD patients (all female: age 77, 82, 86,87, 91, 91) were given green tea powder $1 \mathrm{~g}$ at every meal for 2 months. Their blood HA level and MMSE score were measured before and after taking green tea powder. The relationship between blood HA level change and MMSE score change was investigated. The strong statistically significant negative relationship between blood HA level change and MMSE score change: $r=-0.96, p=0.00018, n=6$. From our observation, it shows that blood HA level change induced MMSE score change, that is, Alzheimer's cognitive ability was controlled by blood HA level. Now we can present that some healthy food, that is named HBF, can recovered $100 \%$ Alzheimer's cognitive impairment by the decreasing the homocysteic acid in a peripheral blood. We made the open-trial of HBF on the Alzheimer's and lewy bodies dementia's cognitive recovery. Two male lewy bodies dementia patients, 11 female Alzheimer's patients were enrolled. All patients were at end stage. They ingested HBF supplement $1.5 \mathrm{~g}$ at every meals for 1-2 months. Their MMSE scores were measured before and after their HBF ingestion. Their blood homocysteic acid levels were measured by ELISA method before and after their ingestions. Results were extremely amaging. 100\% of all patients showed their cognitive recovery. Their blood homocysteic acid decreased their level by HBF. Now we have made a relative large open trial of AD patients. 91 patients were enrolled. Their cognitive recovery were measured by NM scale (New Clinical Scale for Rating of Mental States). All patients who took HBF showed the recovery of their behaviors. From this open-trial of HBF, many facts can be confirmed. (1) Alzheimer's cognitive impairment can be recovered at even end stage. (2) Alzheimer's disease is induced by homocysteic acid.
\end{abstract}

Key words: Recovery of Alzheimer cognition, homocysteic acid, hydrogen brain food.

\section{Introduction}

Iodine plays a key role in maintaining the normal fuAmyloid beta hypothesis is a central dogma for Alzheimer's pathology. However recent two clinical trials of amyloid treatment (Pfzier and Eli Lilly) were all failed to recover the cognitive impairment $(1,2)$, then we are forced to reconsider the central amyloid hypothesis. What does make the cognitive impairment of Alzheimer's disease?

Recent Alzheimer Disease Neuroimaging Initiative (ADNI) reported that even 20 several years before Alzheimer's disease appear, started the amyloid accumulation in healthy people (3), which means amyloid itself does not show any strong neurological

1. Saga Memorial Hospital, Saga; 2. MRD co.

Corresponding Author: T Hasegawa, Saga Memorial Hospital, Saga, Japan. ghf238134@leo.bbiq.jp toxicity for these brains. However amyloid itself disturbed the cognitive ability in mice (4), then why could amyloid not inhibit the cognition in human? There must be the big discrepancy about the effect of amyloid between human and mice.

In human, there must be other factors which strengthens amyloid toxicity or induces the neurodegeneration by itself. Because why do the accumulated amyloid not show its toxicity before Alzheimer's disease appears? And Alzheimer's disease appearing means that some pathogens other than amyloid induce disease process in human. What are they?

\section{Materials and Methods}

All AD cases met the NINCDS-ADRDA criteria, and the diagnosis was assisted by CT,MRI, and SPECT 
findings. 6 Alzheimer's patients agreed this clinical trial of the ingestion of green tea powder (1g) at every meal for 2 months. The ethics committee of Saga University accepted our clinical trial.

Green tea powder were gifted by Ito co. The composition of green tea powder were $70 \%$ powder and $30 \%$ corn starch.

The $1 \mathrm{ml}$ of blood serum was obtained and blood homocysteic acid was measured by ELISA method.

6 patients stayed in same care house all day long, which means they took the same foods.

Regression analysis was conducted with Microsoft excel method.

All patients accepted this clinical trial with document. Ethics committee of Saga memorial hospital agreed this clinical trial. All patients stay in the senior care house whose medical care were all supported by Saga Memorial hospital.

Table 1

Clinical trial of cognitive recovery withHBF

\begin{tabular}{lllllllll}
\hline Patient No & $\mathbf{1}$ & $\mathbf{2}$ & $\mathbf{3}$ & $\mathbf{4}$ & $\mathbf{5}$ & $\mathbf{6}$ & $\mathbf{7}$ & $\mathbf{8}$ \\
\hline Age & 88 & 84 & 91 & 92 & 73 & 91 & 90 & 75 \\
Gender & M & M & F & F & F & F & F & M \\
Disease & A & L & A & A & A & A & A & L \\
& & & & & & & & \\
Patient No & 9 & 10 & 11 & 12 & 13 & & & \\
Age & 80 & 90 & 75 & 91 & 88 & & & \\
Gender & F & F & F & F & F & & & \\
& A & A & A & A & A & & & \\
\hline
\end{tabular}

Gender:M=Male, F=Female; A:Alzheimer, L:Lewy body dementia

All patients ingested HBF supplement $1.5 \mathrm{~g}$ at every meal for 1-2 months. Their MMSE scores were measured before and after 1-2 months HBF ingestion.

91 patients were enrolled in the open field trial. They took our HBF as consumers and their caregivers and doctors measured their behaviors according to NM scale. After 2 months later, their behaviors were measured and their reports were collected.

\section{Results}

6 Alzheimer's patients were all female. Their ages were 77, 82, 86,87, 91, 91.

Green tea powder induced MMSE change and their blood HA change. The results were shown in Fig. 1. 3 patients showed the decrease change in blood HA (number 1,2,5), but two patients showed the increase (number3,4) and 1 patient showed no change (number 6).

\section{Figure 1}

Effect of green tea powder on the blood HA level and MMSE score

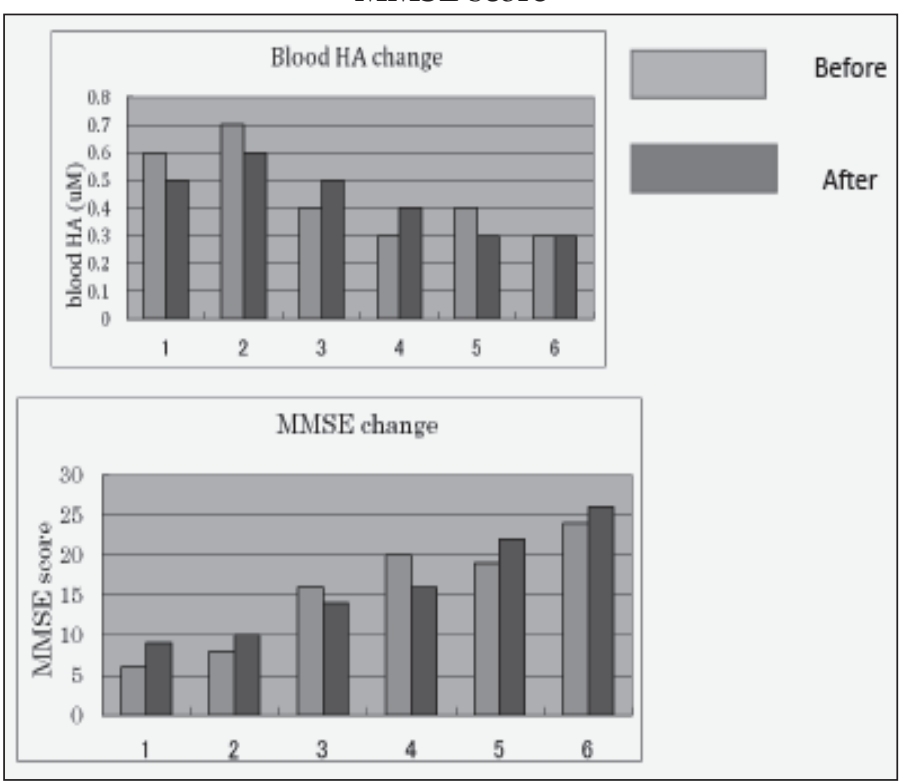

Before: before ingestion of green tea powder. After: 2 months later after the ingestion of green tea powder.

Number shows the Alzheimer's patient. 1: age 82, 2: age 77, 3: age 91 4: age 87,5 : age $91,6:$ age 86

On the other hand, their MMSE score change were as follow:

Number $1: 6$ to 9 , Number 2: 8 to 10 , Number 3: 16 to 14, Number 4: 20 to 16

Number 5: 19 to 22, Number 6: 24 to 26

The relationship between the blood HA change and MMSE score change.

The result is shown in Fig. 2. Clearly shown in Fig. 2 , the relationship between blood HA change (percent change) and MMSE score change is strong negative statistical significant $(\mathrm{r}=-0.96, \mathrm{p}=0.00018, \mathrm{n}=6)$.

Figure 2

Change relationship between HA and MMSE

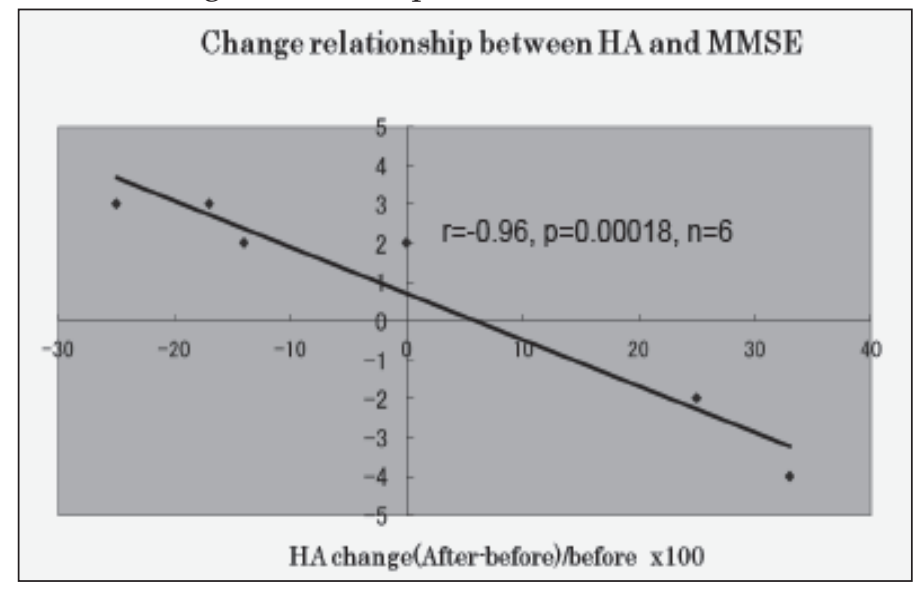

Blood HA level change is shown as (after-before)/ before x 100, MMSE score change is shown as after-before 
Fig. 3 shows the relationship between blood HA level and MMSE score in 12 cases. The strong negative statistical significant was observed between blood HA level and MMSE score $(\mathrm{r}=-0.94, \mathrm{p}=0.00000642, \mathrm{n}=12)$

Figure 3

The relationship between blood HA level and MMSE score

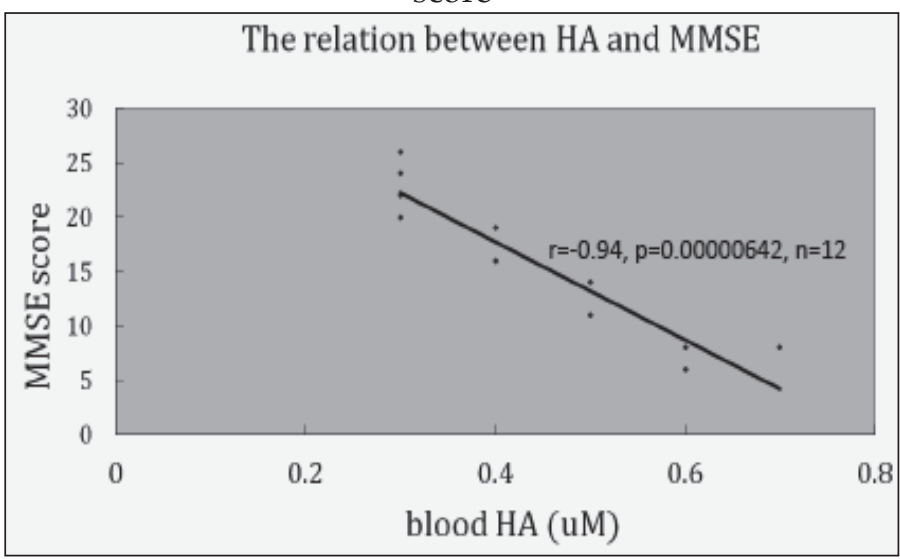

Data was obtained from Fig. 1

\section{Clinical Trial}

Lewy body dementia, 2,8 were all at end stage. No 2 was almost danger to die. He could not communicate to their families. They showed daytime drowsiness all day long. He shows the swallowing difficulty. They were suffering from visual hallucination. However they (2, 8 ) are now very active and they enjoy their lives. First they can communicate to their families. No 2 swallowing difficulty completely disappeared. Their daytime drowsiness completely disappeared. One patient (2) can play harmonica at present. No 8 patient, he could not calculate at all. But now he can calculate and he shows normal brain function. His home doctor (8) has shocked at his recovery. Their families are very thankful for our HBF efficacy. Their visual hallucinations disappeared completely at present.

Other Alzheimer's patients increased their cognitive ability with time.

Especially I have observed these patients blood homocysteic acid has been decreased by HBF. (Fig. 5)

Their MMSE scores were measured before ingestion. 1 month and 2 months later ingestion of HBF, MMSE score were measured. All patients' MMSE score increased by HBF. (Fig. 4)

On the other hand, control did not show any change of their MMSE score with time. (data are not shown here).

91 patients behaviors were measured according to NM scale by their caregivers and doctors. As shown in Fig. 6 , their behaviors assessments were clearly increased as compared before $\mathrm{HBF}$ ingestions.

These results of 91 patients were summarized in table 2. Final stage of patients recoverd their cognitions to that of moderated stage, moderate stage of patients recovered to that of normal stage.

Figure 4

Effect of HBF on MMSE score

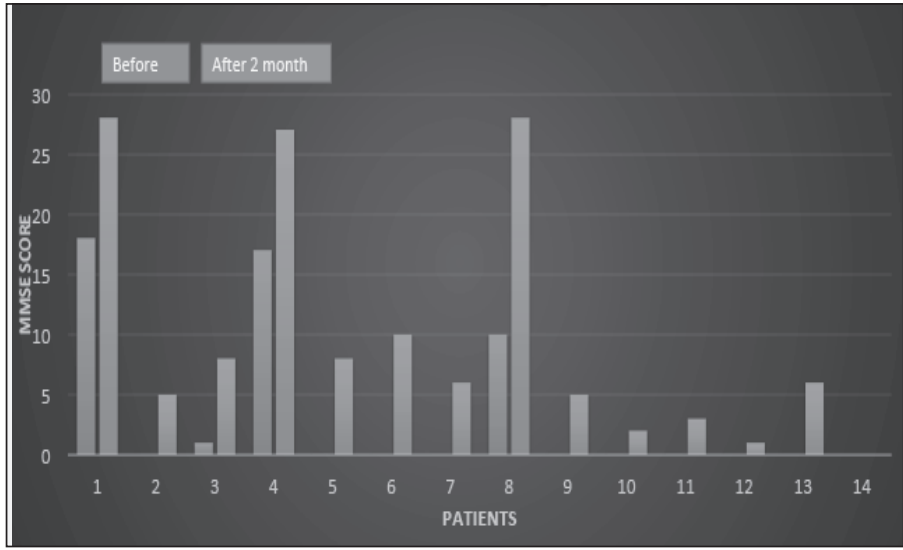

Blue bar:before ingestion of HBF, Brown bar:one month later, Green bar:2 months later. Pateints No,2,5,6,7,9-13 are all MMSE score zero before ingestion of HBF.

Figure 5

Effect of HBF on blood homocysteic acid level

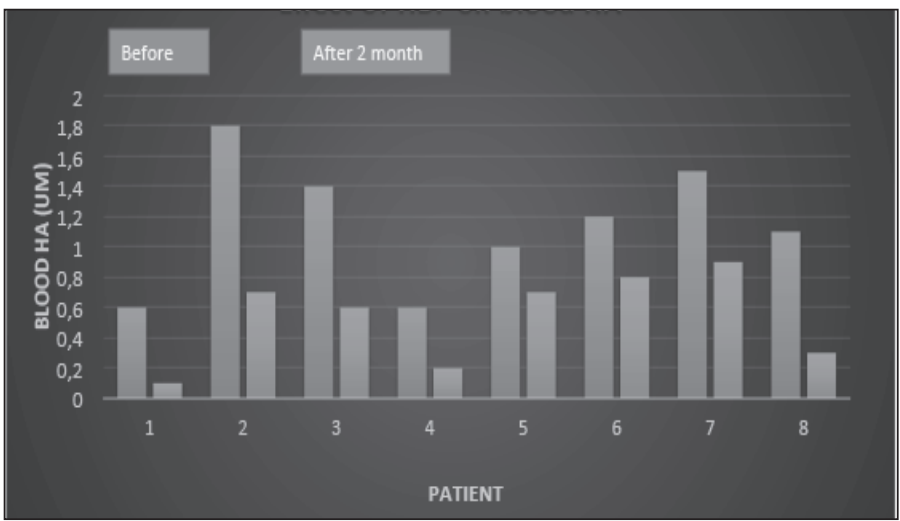

HA measurement was conducted by ELISA method.

Figure 6

Open field trial of HBF for AD patients

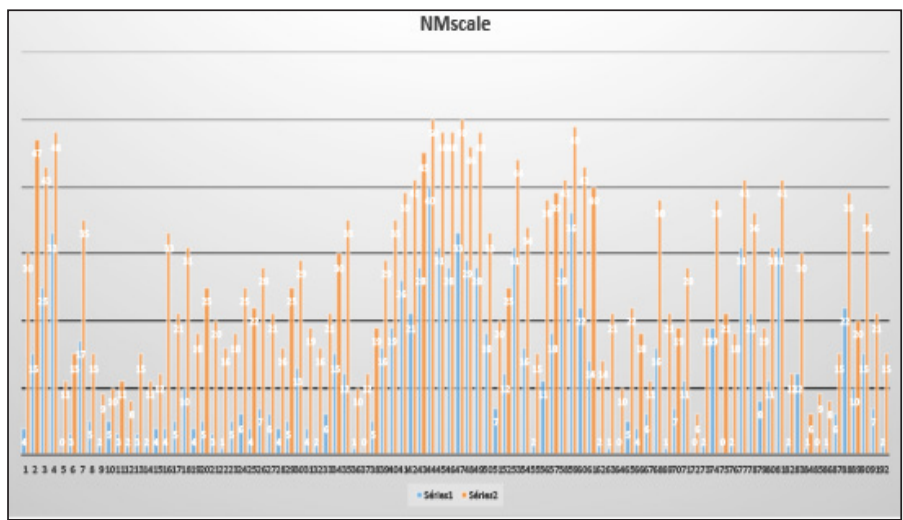

All patients were checked by doctors and we got their agreements for assessment of their behaviors by NM scale; Blue bar indicates before HBF ingestion and yellow bar indicates 2 months later HBF ingestions; Score:0-16: final stage, 17-30:middle stage, 31-42 early stage, 43-50 normal 
Table 2

Effect of green tea powder on urinary HA level

\begin{tabular}{lccc}
\hline & Before & After & $\%$ inhibition \\
\hline No 1 & 14.9 & 5.1 & 66 \\
No 2 & 62 & 26.8 & 57 \\
No 3 & 50 & 43.7 & 13 \\
No 4 & 0.5 & 0.4 & 20 \\
No 5 & 21.8 & 11.7 & 50 \\
No 6 & 20.8 & 8.5 & 60 \\
\hline
\end{tabular}

Urinary HA level: $\mu$; HA measurement was conducted by ELISA method

\section{Discussion}

This time all volunteers are Alzheimer's patients and all female. Their ages were between 71 and 91. However they stayed all day long in same care house, which indicates they took same foods. The green tea powder affected blood HA level by itself, not by foods. The blood HA level is affected by the kidney function. From our previous observation, the blood HA was originated from urine (5). Usually HA is excreted into urine and the blood HA is not detectable. But urinary HA excretion is inhibited by aging, the blood HA is detected. The green tea powder inhibits the HA production in kidney by their antioxidant action.

Urinary HA levels in number 1,2,5,6 were decreased by $50-56 \%$ by green tea powder. However HA level in urine in number 3,4 were decreased by only 13-20\%. (Table 1 ). So their blood HA level did not decrease and increased its level after ingestion of green tea powder.

We observed the relationship between blood HA level and MMSE score.

Fig. 3 shows its relationship. It shows the almost perfect relationship.

And Fig. 2 shows the very strong perfect relationship between blood HA level change and MMSE score change. From Fig. 2 result, we can say that blood HA can control the MMSE score change. That is, blood HA can control the cognitive ability in Alzheimer's patient.

Why can the blood HA control cognitive ability in patients?

First HA is a glutamate neurotransmitter and HA itself can induce neurodegeneration at a higher level with no amyloid (6), and the HA toxicity induces a calcium influx (7) or a mitochondrial inhibition (8) or oxidative stress (6) to bring the neuronal disfunction.

NMDA receptor in blood brain barrier $(\mathrm{BBB})$ can cotrol its permeability $(9,10)$. Then the blood HA can increase the permeability of $\mathrm{BBB}$ and $\mathrm{HA}$ can pass through $\mathrm{BBB}$ and enters into hippocampus, because hippocampus's $\mathrm{BBB}$ is very thin (11). And entered HA can interact with already accumulated amyloid beta and HA increases its neurotoxicity, because amyloid beta increases HA toxicity
(12).

Now we can explain why the accumulated amyloid beta does not show the neurotoxicity. Amyloid beta is already accumulated in healthy brain before Alzheimer's disease starts (3). This phenomenon is similar to that of mice. Mice do not show any neurodegeneration in the presence of accumulated amyloid. In human case, just memory is impaired similar as that of mice. The blood HA is not detected before AD appear in human. However the blood HA level increases its level, then the interaction between HA and amyloid beta reveals HA toxicity and the neurodegeneration starts.

The discrepancy of Alzheimer's pathogenic process between human and mice may be HA pathogen, because mice does not inhibit urinary excretion of HA with aging (unpublished observation). However human decreases urinary HA excretion with aging. Then mice do not show HA toxicity in usual state. However presenilin gene activated mice show HA toxicity, because presenilin activates calcium flow (14) and calcium activates cystathione beta-synthase (15) which catalyzes HA production (unpublished observation). Then amyloid does not induce the neurodegeneration in usual mice without HA.

And this Alzheimer's neurodegeneration is strongly related to aging in human. Why is the aging related to Alzheimer's disease?

But amyloid accumulation cannot induce the neurodegeneration and is not related to aging. Then what is related to aging in human?

Human shows the HA toxicity with aging which induces kidney disfunction and urinary HA excretion is inhibited to induce the blood HA.

Recently it has been reported that APOE4 disrupted the permeability of blood brain barrier in human (16), then blood HA can easily enter into brain of APOE4 carrier who is well-known as earlier AD starting than non APOE4 carrier.

Then it is well-known that an exercise prevents Alzheimer's disease (12) and or cures the cognitive impairment, because exercise can induce activation of kidney function to decrease the blood HA level.

Finally amyloid hypothesis is still active and HA toxicity should be considered in human Alzheimer's disease process with amyloid hypothesis.

Now we discuss about this clinical trial. First we observed the advanced stage of patients could be cured and increased their cognitive ability. In general the advanced stage of Alzheimer's disease shows the neuronal degeneration and cannot increase their cognitive ability. However we could observe the increase of their cognitive ability. Why could we observe this increase? One evidence supports that our observation for the decrease in blood homocysteic acid could be confirmed by HBF (Fig. 5). Why could HBF decrease in blood homocysteic acid? HBF induced the active hydrogen generation in blood, which could decompose 
sulfonic group of homocysteic acid by hydrogen reduction. This active hydrogen reduction for the sulfonic group of homocysteic acid is so strong that the pathogenic homocysteic acid can be disappeared and consequently the cognitive ability increases (Fig. 2).

Now we can confirm by our clinical trial of HBF that Alzheimer's disease can be recovered their cognitive ability by the decrease in blood homocysteic acid.

Especially our open field trial confirms our hypothesis, because so many patients (91 patients) could recover their cognitions according to our NM scale measurements. Our open field trial showed the recovery of final stage of AD. This recovery cannot be observed by any drugs, any treatments.

Our HBF supplement can only recover the final stage of AD patients cognitions. Of course, moderate stage of patients recovered their cognitions to that of normal. It is very important observations. Our HBF can recover Alzheimer's cognitions and we can overcome Alzheimer's disease.

However we should admit that our open field trial has a problem, because their measurement of NM scale has uneaqual measurement.

Objective evaluation is difficult for measurement and if anything there is measurement by a wrong impression.

Acknowledgement: The author (H.T) thanks Ito Co. for supporting this clinical observation by their financial supports and by their proving with green tea powder.

Ethical Standards: Saga Memorial Hospital ethical committee accepted this clinical observations.

Conflicts of Interest: All authors have no conflict of interest.

\section{References}

1. Bapineuzumab Phase 3: Target Engagement. But No Benefit. Alzheimer Resarch Forum 12 September 2012. Available at http:/ / www.Alzforum.org/ new $/$ detail.asp?id=3268

2. The Solanezumab Benefit: Oh. So Small, But Probably Real. Alzheimer Research Forum 9 October 2012 Available at http://www.Alzforum.org/ new $/$ detail.asp?id=3288
3. API Echoes DIAN: Biomarker Changes Precede Symptoms by 20 years. Alzheimer Research Forum 8 November 2012. Available at http:/ /www. Alzforum.org/new / detail.asp?id=3311

4. Kawahara K, Suenobu M, Yoshida A, Koga K, Hyodo A, Ohtsuka H, Kuniyasu A, Tamaamki N, Sugimoto Y, Nakayama H. Intracerebral microinjection of interleukin-4/interleukin-13 reduces $\beta$-amyloid accumulation in the ipsilateral side and improves cognitive deficits in young amyloid precursor protein 23 mice. Neurosci. 2012;207, 243-260

5. Hasegawa, T., Mikoda, N., Kitazawa, M., Laferla, FM. Treatment of Alzheimer's Disease with Anti-Homocysteic Acid Antibody in 3xTg-AD Male Mice. PLoS ONE 5(1): e8593. doi:10.1371/journal.pone.0008593

6. Lockhart B, Jones C, Cuisinier C, Villain N, Peyroulan D, et al. Inhibition of L-homocysteic acid and buthionine sulphoximine-mediated neurotoxicity in rat embryonic neuronal cultures with a-lipoic acid enantiomers. Brain Research 2000; 855(2): 292-297.

7. Shankar GM, Bloodgood BL, Townsend M, Walsh DM, Selkoe DJ, et al. Natural Oligomers of the Alzheimer Amyloid-s Protein Induce Reversible Synapse Loss by Modulating an NMDA-Type Glutamate ReceptorDependent Signaling Pathway. J Neurosci 2007 27(11): 2866-2875.

8. Folbergrova' J, Jes`ina P, Drahota Z, Haugvicova' VL, Vojtı's`kova' A, et al. Mitochondrial complex I inhibition in cerebral cortex of immature rats following homocysteic acid-induced seizures. Experimental Neurology 2007;204(2): 597-609

9. Ramos Coutinho M, da Silva LF, Menescal-de-Oliveira L. Modulation of tonic immobility in guinea pig PAG by homocysteic acid, a glutamate agonist. Physiol Behav. 2008;94, 468-473

10. Beard RS Jr, Reynolds JJ, Bearden SE. Hyperhomocysteinemia increases permeability of the blood-brain barrier by NMDA receptor-dependent regulation of adherens and tight junctions. Blood 2011;118, 2007-2014

11. Vorbrodt AW, Dobrogowska DH, Ueno M, Tarnawski M. A quantitative immunocytochemical study of blood-brain barrier to endogenous albumin in cerebral cortex and hippocampus of senescence-accelerated mice (SAM). Folia Histochem Cytobiol. 1995;33, 229-237

12. Hasegawa T, Ukai W, Jo DG, Xu X, Mattson MP, Nakagawa M, et al Homocysteic Acid Induces Intraneuronal Accumulation of Neurotoxic Ab42: Implication for the Pathogenesis of Alzheimer's disease. J Neurosci Res 2005;80: 869-876.

13. Neely Kayala KM, Dickinson GD, Minassian A, Walls KC, Green KN, Laferla FM. Presenilin-null cells have altered two-pore calcium channel expression and lysosomal calcium: Implications for lysosomal function.Brain Res. 2012;1489, 8-16

14. Qu K, Lee SW, Bian JS, Low CM, Wong PT. Hydrogen sulfide: neurochemistry and neurobiology. Neurochem Int. 2008;52, 155-165

15. Thomas O. Obisesan, Richard F. Gillum, Stephanie Johnson, Nisser Umar, Deborah Williams, Vernon Bond, and John Kwagyan. Neuroprotection and Neurodegeneration in Alzheimer's Disease: Role of Cardiovascular Disease Risk Factors, Implications for Dementia Rates, and Prevention with Aerobic Exercise in African Americans. Int J Alzheimers Dis. 2012; 2012: 568382. Published online 2012 April 17. doi: 10.1155/2012/568382

16. Sperling R, Salloway S, Brooks DJ, Tampieri D, Barakos J, et al. Amyloidrelated imaging abnormalities in patients with Alzheimer's disease treated with bapineuzumab: a retrospective analysis. Lancet Neurol. 2012 Mar;11(3):241-9. doi: 10.1016/S1474-4422(12)70015-7. Epub 2012 Feb 